\title{
NEGLECTED DISEASES AND RADIATION
}

The Journal of Venomous Animals and Toxins including Tropical Diseases publishes works involving tropical diseases, toxinology, venoms and their derivatives. According to the World Health Organization, tropical diseases are considered neglected, and only one percent of the new drugs developed by the private industry are geared to them. The fact that these diseases are not a Public Health problem in developed countries may justify this indifference. However, millions of people all over the world are at risk of contracting these diseases, and, annually, millions of deaths, especially of children, are attributed to them.

For example, after almost a century from Chagas disease discovering, there is not an efficacious treatment available for the population chronically infected neither a perspective of developing a vaccine for it. This situation is the same for Leishmaniasis, Toxoplamasis, Malaria, and other tropical diseases, which have become less frequent in big centers but still insist in causing epidemic outbreaks, showing the maintenance of their causing factor. Despite these aspects, South American researchers, mainly from Brazilian Universities and Research Institutes, have been making great efforts to correct these failures, trying to eradicate these diseases or at least develop preventive methods.

For ophidic accidents, whose serum therapy is still the only efficacious treatment, the search lies in obtaining vaccines and making serum available for veterinary use. This state of affairs has stimulated researchers to seek alternatives in order to achieve good results in this arduous task.

The use of ionizing radiation has been decisive in the obtainment of modified proteins with particular and promising properties, both from venoms and parasites. This radiation has already proved to reduce the toxicity of venoms or toxins isolated from snakes, spiders, scorpions, and bees, maintaining or even improving their immunological properties. This is due to the radiation ability to modify proteins, making them easier recognized by the immune system, improving thus the vaccines developed from antigens subjected to this process. This was particularly demonstrated by our group, both in venom proteins, like crotoxin, and in the increase of immunogenicity of recombinant proteins from Mycobacterium leprae, Hansen disease bacillus.

Another important aspect of ionizing radiation is its ability to sterilize without decreasing the viability of protozoans, such as malaria and toxoplasmosis. This has allowed the production of promising vaccines, both for irradiated sporozoites and taquizoites. This capacity of sterilizing without killing, i.e., only thwarting reproduction while keeping the agent's cellular biology's properties and capacity of invasion, along with the same kind of exposal by natural infection antigens, is ideal for vaccine production.

This is simpler and so efficient as the production of genetically modified protozoans, recently described to have the same ability.

Exploiting these aspects better can enhance our ability to produce vaccines, not only in these mechanisms but also in the selection of metacycle forms of kinetoplastids, which are difficult to obtain in axenic cultures.

The association of ionizing radiaton with tropical or neglected diseases is very interesting. Radiation was always stigmatized for its association with the damage caused by bombs and nuclear accidents, nevertheless its pacific use is extremely beneficial. Sterilization by radiation of plastic products has enabled widening of their use in Medicine and Biology. Certainly, new pacific uses are going to be discovered. The association of the use of radiation for production of vaccines and immunogens, aiming at preventing tropical diseases and accidents with venomous animals, is only one of its possible applications. Indubitably, our researchers will not lack the ingenuity to use it in other systems that aim at decreasing these social issues which are tropical diseases.

Nanci do Nascimento

Pesquisadora do Instituto de Pesquisas Energéticas e Nucleares - IPEN/CNEN - São Paulo. Email: nnascime@ipen.br 